\title{
First reports of adverse drug reactions and interactions
}

C) Springer Nature Switzerland AG 2020

Table 1 contains an overview of first published case reports of adverse drug reactions and interactions identified in the international literature in recent weeks by Reactions Weekly, the Adis drug safety newsletter. Reactions Weekly provides summaries of adverse drug reaction news sourced from journals, scientific meetings, media releases, regulatory agency websites, and bulletins from the National Centers that participate in the WHO International Drug Monitoring Programme.

\begin{tabular}{|c|c|}
\hline Drug: adverse drug reaction & References \\
\hline $\begin{array}{l}\text { Corticosteroids (prednisolone and hydrocortisone): reactivations of } \\
\text { Paracoccidioidomycosis pneumonia (serious) }\end{array}$ & $\begin{array}{l}\text { Healey S, et al. First report paracoccidioidomycosis reactivation as a } \\
\text { complication of immunosuppressive therapy for acute severe colitis in a } \\
\text { caving enthusiast. BMJ Case Rep. 2020;13(7) }\end{array}$ \\
\hline Diltiazem: acute pharyngeal inflammatory mass (serious) & $\begin{array}{l}\text { Manning W, et al. Acute airway inflammation caused by incomplete } \\
\text { ingestion of extended-release diltiazem: a case report. J Emerg Med. } \\
\text { 2020; 58(6):e247-e249 }\end{array}$ \\
\hline Durvalumab: phimosis (serious) & $\begin{array}{l}\text { Vazquez-Lavista LG, et al. Phimosis: a rare complication of immuno- } \\
\text { therapy with durvalumab. Urol Case Rep. 2020;33:101350 }\end{array}$ \\
\hline Gliquidone and dulaglutide: QT prolongation & $\begin{array}{l}\text { Ghafari C, et al. Exercise-induced torsades de pointes as an unusual pres- } \\
\text { entation of cardiac sarcoidosis: a case report and review of literature. } \\
\text { World J Cardiol. 2020;12(6):291-302 }\end{array}$ \\
\hline Infliximab: delayed infusion reaction in the form of lactic acidaemia & $\begin{array}{l}\text { Vigh N, et al. Lactic acidemia due to an infliximab infusion reaction. Am } \\
\text { J Emerg Med. 2020;38(4):850.e1-850.e3 }\end{array}$ \\
\hline Mirtazapine: prostatism & $\begin{array}{l}\text { Gundogmus I, et al. Mirtazapine-induced prostatism. Am J Ther. 2020; } \\
\text { https://doi.org/10.1097/MJT.0000000000001140 }\end{array}$ \\
\hline Natalizumab: subacute cutaneous lupus erythematous (serious) & $\begin{array}{l}\text { Bolton C, et al. The first case of natalizumab-induced subacute cutaneous } \\
\text { lupus erythematosus. Rheumatology. 2020;59(6):1446-7 }\end{array}$ \\
\hline Sodium polystyrene sulfonate: rectovaginal fistula (serious) & $\begin{array}{l}\text { Martin LE, et al. Rectovaginal fistula induced by Resonium A: a first case } \\
\text { report and literature review. ANZ J Surg. 2019;89(11):E550-1 }\end{array}$ \\
\hline Tenofovir disoproxil fumarate: tooth loss (serious) & $\begin{array}{l}\text { Jianfei L, et al. Tooth loss associated with the use of tenofovir disoproxil } \\
\text { fumarate. J Clin Pharm Ther. 2020. https://doi.org/10.1111/jcpt.13219 }\end{array}$ \\
\hline
\end{tabular}

An event is serious (US FDA MedWatch definition) when the patient outcome is death, life threatening, hospitalization, disability, congenital anomaly or requires intervention to prevent permanent impairment or damage 